\title{
Geschichte und Entwicklung der Universitäts-Hautklinik Homburg/Saar
}

\author{
Development of the Department of Dermatology at the Saarland University Hospital
}

Autoren

Institut

\section{S. Psaier, K. Rass}

Klinik für Dermatologie, Venerologie und Allergologie, Universitätsklinikum des Saarlandes

\section{Bibliografie}

Dol http://dx.doi.org/

$10.1055 / \mathrm{s}-0030-1256108$

Akt Dermatol 2011; 37:

31-37 @ Georg Thieme

Verlag KG Stuttgart · New York

ISSN 0340-2541

Korrespondenzadresse

Sigrid Psaier

Klinik für Dermatologie, Venerologie und Allergologie Universitätsklinikum des Saarlandes

Kirrbergerstr. 1 - Gebäude 18 66421 Homburg sigrid.psaier@uks.eu

\section{Zusammenfassung $\nabla$}

Im Jahr 2009 feierte das Universitätsklinikum des Saarlandes in Homburg sein 100-jähriges Bestehen. Bereits 1945 wurde eine Abteilung für Hautund Geschlechtskrankheiten im Landeskrankenhaus Homburg eingerichtet, welche 1946 nach der Inneren Medizin die zweitgrößte Bettenzahl

\section{Einleitung}

\section{$\nabla$}

Das Universitätsklinikum des Saarlandes in Homburg/Saar blickt heute auf eine 100-jährige Geschichte zurück. Zwischen dem Gründungsjahr 1909 und heute liegt ein bewegtes Jahrhundert, welches im Saarland im Zuge zweier Weltkriege und zweier leidenschaftlich diskutierter Volksabstimmungen, 1935 und 1955, vor allem auch durch den Wechsel verschiedener politischer Systeme geprägt war: das Ende des Wilhelminischen Kaiserreichs im Ersten Weltkrieg, die Zeit der 15jährigen Völkerbundverwaltung, zehn Jahre NSDiktatur mit dem Zweiten Weltkrieg, der Aufbruch zum teilautonomen Saarland nach dem Krieg und schließlich 1957 der politische Beitritt zur Bundesrepublik Deutschland. In diese turbulente Zeit Anfang und Mitte des vergangenen Jahrhunderts fielen der Aufbau und die frühe Entwicklung der Klinik für Haut- und Geschlechtskrankheiten der Universität des Saarlandes und zeitgleich die Entfaltung der Universität des Saarlandes selbst.

\section{Pfälzische Heil- und Pflegeanstalt und Landeskrankenhaus Homburg (1909-1947)}

1909 wurde auf dem heutigen Campus des Klinikums nach Frankenthal und Klingenmünster die dritte Kreisirrenanstalt auf dem Boden der Baye- des Klinikums aufwies. Die heutige Klinik für Dermatologie, Venerologie und Allergologie des Universitätsklinikums verfügt über die moderne und umfassende Infrastruktur eines Krankenhauses der Maximalversorgung. Der Artikel soll Einblick in die wichtigsten Entwicklungsschritte des Klinikums, von seiner Gründung bis zur Gegenwart, geben.

rischen Pfalz als „Pfälzische Heil- und Pflegeanstalt“ eröffnet. Nach den Plänen des Regierungsund Bauassessors Heinrich Ullmann entstand auf einer Fläche von 400 Hektar das bis heute im Grunde erhalten gebliebene Pavillonsystem mit damals zunächst 49 Gebäuden mit vielen Elementen des Jugendstils ( $\bullet$ Abb. 1, 2).

Die 24 Krankenpavillons waren für die Aufnahme von bis zu 1000 „geisteskranken“ Patienten konzipiert. Quellen zur inneren Organisation und Administration der Einrichtung, zum Profil der Ärzteschaft, zu Zahl, regionaler und sozialer Herkunft und zu den Krankheitsbildern der Patienten sowie zu angewandten Therapieverfahren sind leider nicht erhalten geblieben. Mit Ausnahme der belegbaren Umnutzung für Lazarette im Ersten Weltkrieg lässt sich die Entwicklung der Heilund Pflegeanstalt Homburg während der Kriegsjahre, der anschließenden französischen Besatzung und der territorialen Veränderungen mit einem neu geschaffenen „Saargebiet“ unter Völkerbundverwaltung bis zu Beginn der 1920er-Jahre ebenfalls nur schwer rekonstruieren [1]. Infolge der politischen Veränderungen nach 1918 und des Versailler Vertrages mit der Gründung des „Saargebiets“ unter Völkerbundverwaltung (1920-1935) wurde die Heil- und Pflegeanstalt auf Beschluss der Regierungskommission des Saargebietes in ein Landeskrankenhaus $(\bullet$ Abb. 3) umgewandelt, welches künftig die medizinische Versorgung der Region gewährleisten sollte. 


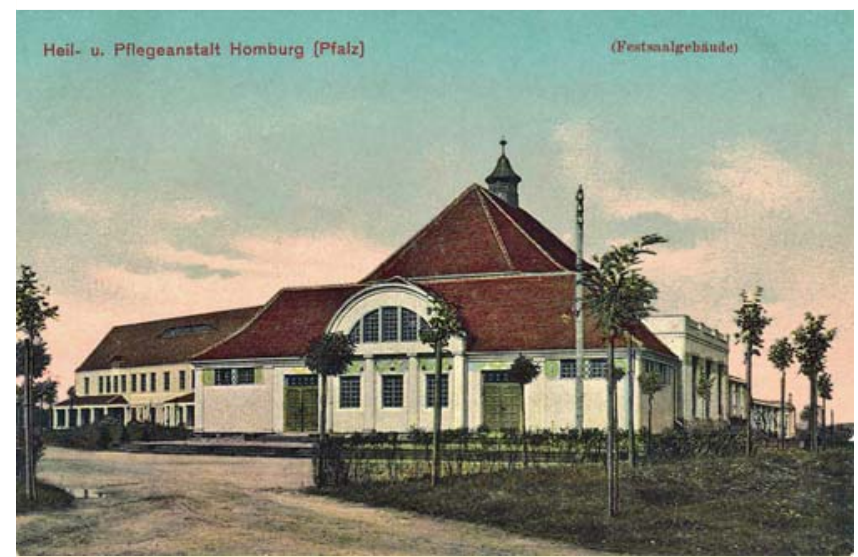

Abb. 1 Die Pfälzische Heil- und Pflegeanstalt Homburg, Festsaalgebäude. Heute befindet sich hier die Universitätsbibliothek der Medizinischen Fakultät.

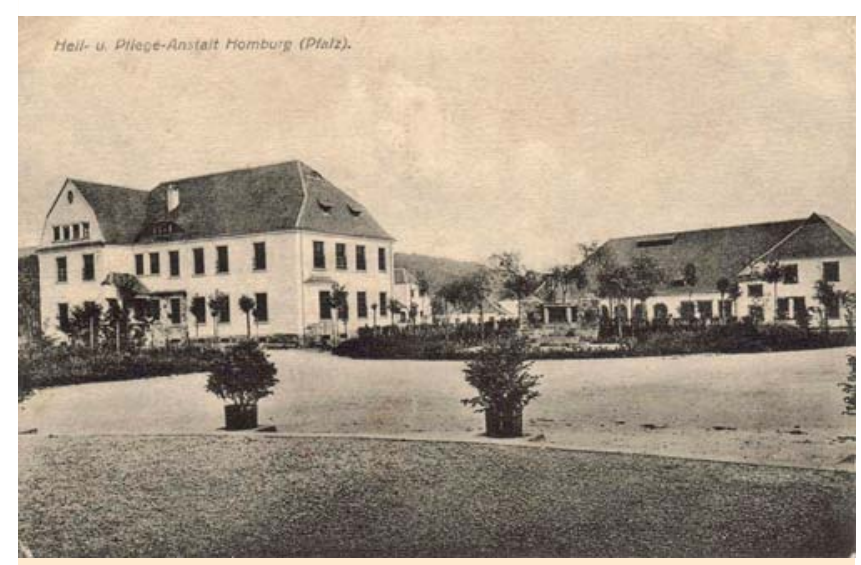

Abb. 2 Die Abbildung zeigt auf der linken Bildhälfte das spätere Gebäude 35 der Homburger Hautklinik zu Zeiten der Pfälzische Heil- und Pflegeanstalt.

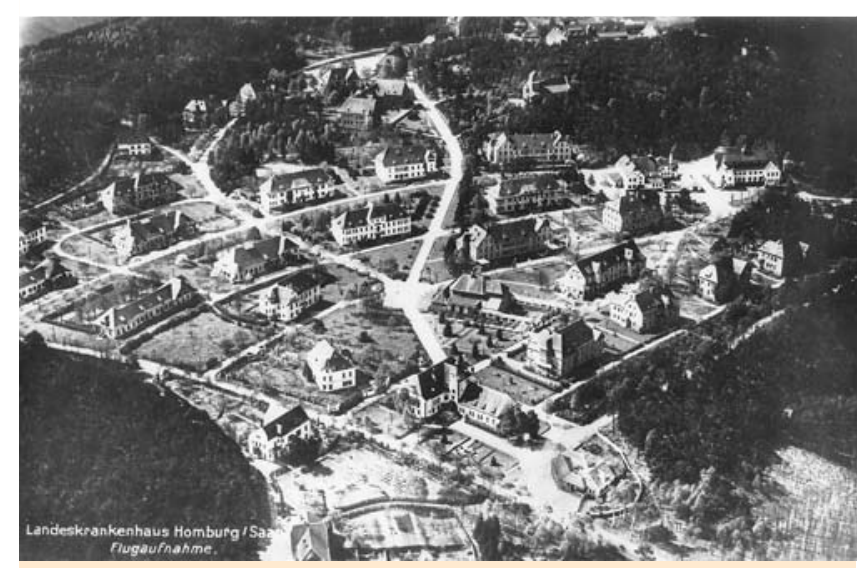

Abb. 3 Übersicht über das Gelände des Landeskrankenhauses Homburg.

Im Herbst 1921 wurden die ca. 450 verbliebenen „geisteskranken“ Patienten aus der Pfalz in die pfälzische Heil- und Pflegeanstalt Klingenmünster und die aus dem neuen Saargebiet stammenden Patienten in die ehemals preußische Anstalt Merzig überwiesen. Mit der Einrichtung des Landeskrankenhauses 1922 begann somit die neue Ära der medizinischen Entwicklung zum universitären Klinikum des Saarlandes. Primär wurden Fachabteilungen für Chirurgie/Gynäkologie, Orthopädie, Innere Medizin, Abteilungen

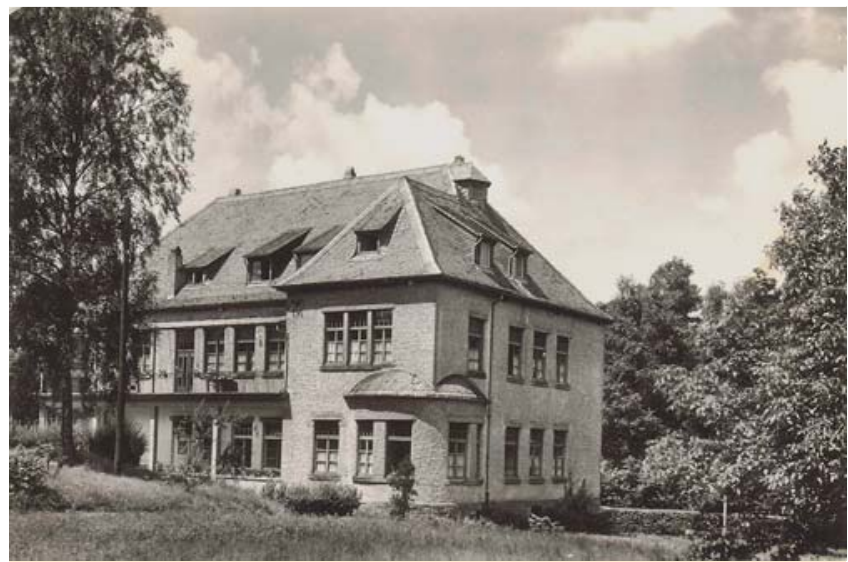

Abb. 4 Rückwärtige Ansicht von Gebäude 35 (Bettenhaus und Direktorium) der Homburger Hautklinik nach dem Zweiten Weltkrieg.

für Lungenkranke, Nervenkranke, eine Säuglings- und Kinderabteilung und eine geburtshilfliche Abteilung mit insgesamt 680 Betten etabliert. Unter der ärztlichen Leitung von Prof. Oskar Orth, der diese Funktion von 1922 bis zu seiner Emeritierung 1947 innehatte, wurden hier bereits regelmäßig wissenschaftliche Fortbildungen und medizinische Demonstrationen veranstaltet.

Nach der Volksabstimmung vom 13.1.1935 wurde das Saargebiet in das nationalsozialistische Deutschland eingegliedert. Während der Zeit des NS-Regimes wurden auf der Basis der Rassenideologie der NSDAP im Zeitraum 1935-1939 Zwangssterilisationen von „Erbkranken“ auch im Landeskrankenhaus Homburg durchgeführt. Zu Beginn des Zweiten Weltkrieges erfolgte dann die „kriegsbedingte Räumung“ des Landeskrankenhauses zugunsten einer militärischen Nutzung. Es ist belegt, dass im Zuge dieser Räumung ein großer Teil der psychiatrischen Patienten dem Euthanasieprogramm des NS-Regimes zum Opfer fiel. Während des Zweiten Weltkriegs diente das Landeskrankenhaus in erster Linie als Reservelazarett. 1942 wurden zudem mehrere Baracken für sowjetische Kriegsgefangene und Zwangsarbeiter auf dem Krankenhausgelände errichtet. Für zivile Kranke standen nur noch 248 Betten zur Verfügung, vor allem für Tuberkulosekranke, neurologische und internistische Fälle [2,3].

Am 21. März 1945 erreichten die ersten amerikanischen Soldaten das Homburger Klinikgelände, am 10. Juli 1945 wurde die amerikanische Besatzung jedoch von der französischen Militärverwaltung des Saargebiets abgelöst. Nach Beendigung des Zweiten Weltkriegs wurde das Saarland erneut wirtschaftlich an Frankreich angegliedert. Es bestand ein dringender Bedarf an Krankenhausbetten für ausländische Rückwanderer und zunehmend für erkrankte deutsche Heimkehrer aus den Gefangenenlagern der Alliierten. Daher wurden die Abteilungen des ehemaligen Landeskrankenhauses wieder in Betrieb genommen. 1945 wurde erstmals eine eigenständige Abteilung für Haut- und Geschlechtskranke eingerichtet und somit der Grundstein für die aktuelle Klinik für Dermatologie, Venerologie und Allergologie gelegt ( $\bullet$ Abb.4).

Das genaue Datum der Eröffnung der Hautklinik ist heute nicht mehr bekannt, auch nicht die Namen der ersten Ärzte, die dort tätig waren. Überliefert ist, dass die Hautklinik zu Beginn über 60 Betten verfügte. Der erste namentlich bekannte leitende Dermatologe an der Homburger Hautklinik war Professor Rudolf Strempel $(\bullet$ Abb. 5, $\bullet$ Tab. 1). 


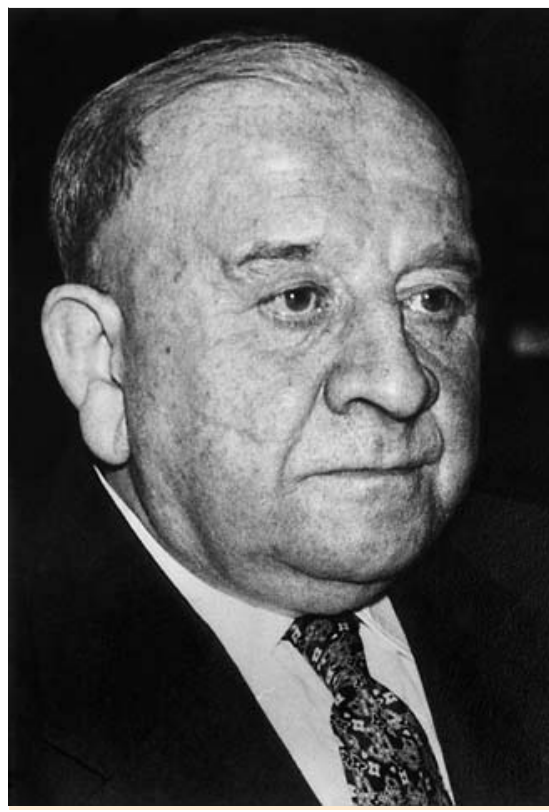

Abb. 5 Rudolf Strempel (1891-1981): Der erste Ordinarius für Dermatologie und Venerologie an den Universitätskliniken des Saarlandes wirkte von $1946-1960$.

Im Januar 1946 wurde durch die französische Militärregierung mit maßgeblicher Unterstützung des Leiters der Abteilung „Öffentliche Gesundheit“ Medécin-Colonel Dr. René Springer genehmigt, dass am Landeskrankenhaus Homburg studienmäßig gegliederte Fortbildungskurse für aus dem Krieg zurückgekehrte saarländische Medizinstudenten durchgeführt werden konnten. Dies ergab sich einerseits aus dem damals erheblichen Mangel an ärztlicher Versorgung und aus der Tatsache, dass die Studenten aus dem Saargebiet an deutschen Hochschulen wegen der überall begrenzten Zahl an Studienplätzen nicht aufgenommen wurden. Die fehlende Anerkennung der Kurse in der französischen Besatzungszone durch deutsche Universitäten führte unterstützt durch das Engagement des französischen Militärgouverneurs Gilbert Grandval im März 1947 zum Anschluss des Landeskrankenhauses an die Universität Nancy.

\section{Gründung der Universitätskliniken des Saarlandes - Ära Prof. Strempel (1946 - 1960)}

Durch die Gründung des Homburger Hochschulinstituts als „Institut d'Etudes Supérieures de l'Université de Nancy en Territoire Sarrois“ mit offizieller Einweihung am 8. März 1947 wurde die Pfälzische Heil- und Pflegeanstalt Homburg schließlich formal aufgelöst. Die Ausbildung in den vorklinischen und theoretischen Fächern erfolgte zunächst durch Professoren aus Nancy, während die klinischen Fächer durch die Leiter der Homburger Kliniken in Deutsch gelesen wurden.

Ab Februar 1948 wurde im Saarland an vier Fakultäten gelehrt, wobei die Medizinische Fakultät eben geschichtlich begründet weiter im kleinstädtischen Homburg verblieb, während die Fakultäten der Philosophie, Rechts- und Naturwissenschaften im Herbst 1948 bzw. 1950 in Saarbrücken, der Landeshauptstadt, angesiedelt wurden. Am 1. Oktober 1948 erfolgte die Konstituierung der Medizinischen Fakultät. Dieses Datum gilt heute auch als Gründungsdatum der Universität des Saarlandes.

Die Universitätskliniken erhielten das Nutzungsrecht für Grundbesitz und Gebäude der früheren Heil- und Pflegeanstalt, blieben jedoch als „Universitätskliniken im Landeskrankenhaus Homburg (Saar)“" ohne eigene Rechtspersönlichkeit und wurden spä-
Tab. 1 Direktoren der Universitäts-Hautklinik in Homburg.

\begin{tabular}{ll} 
Rudolf Strempel & $(1945-1960)$ \\
Fritz Nödl & $(1960-1980)$ \\
\hline Hansotto Zaun & $(1980-1996)$ \\
Wolfgang Tilgen & $(1996-2009)$ \\
Thomas Vogt & (seit 2009)
\end{tabular}

ter - als einzige Universitätskliniken in Deutschland - nicht dem Geschäftsbereich des Wissenschaftsministers zugeordnet, sondern verblieben in der Zuständigkeit des Gesundheitsministers. Diese Regelung führte in der Folgezeit bis 1994 dazu, dass der Krankenversorgung im Homburger Universitätsklinikum - im Vergleich zu anderen deutschen Universitätskliniken - stets ein höherer Stellenwert eingeräumt wurde als den universitären Aufgaben.

Als das Saarland am 1.1.1957 in die Bundesrepublik Deutschland eingegliedert wurde, entsprach das Fächerspektrum der saarländischen Universitätskliniken mit insgesamt elf Einzelkliniken bzw. Klinischen Abteilungen und neun Instituten der Theoretischen oder Klinischen Medizin dem damaligen Stand anderer deutscher Universitätskliniken $[4,5]$.

Im Frühjahr 1946 übernahm Prof. Rudolf Strempel (1891 - 1981) ( Abb.5) die Leitung der neu errichteten Abteilung für Hautund Geschlechtskranke des Landeskrankenhauses Homburg, die er, als gebürtiger Saarländer, bis zum Jahr 1960 leitete. Rudolf Strempel studierte in München und hatte seine dermatologische Weiterbildung bei Erich Hoffmann in Bonn absolviert. 1925 habilitierte er sich mit einer Arbeit über die Serologie der Syphilis. 1930 zum außerordentlichen Professor ernannt, übernahm er 1934 die Leitung des Wehrmachtskrankenhauses Tempelhof in Berlin. Nach ärztlichem Kriegseinsatz, zuletzt als stellvertretender Armeearzt, kehrte er 1946 in das Saarland zurück.

Strempel trug unter den geschilderten schwierigen Bedingungen die Lasten der Aufbauarbeit der Klinik für Haut- und Geschlechtskrankheiten in Homburg. Unter seiner Leitung wurde die Hautklinik von 60 auf 160 Betten erweitert und war damit nach der Inneren Medizin die zweitgrößte Klinik am Campus. Mit einem Anteil von etwa $60 \%$ wurden damals in überwiegender Zahl venerologisch Kranke stationär behandelt (Syphilis, Gonorrhoe), daneben auch ein bemerkenswerter Anteil an Hauttuberkulosen. Strempels wissenschaftliche Tätigkeit konzentrierte sich daher fast ausschließlich auf venerologische Fragestellungen.

Mit Gründung der Universität des Saarlandes wurde Strempel 1950 in den Lehrkörper aufgenommen und zum ersten Ordinarius für Haut- und Geschlechtskrankheiten ernannt.

1957 erhielt die Hautklinik auf seine Bemühungen hin einen eigenen Hörsaal sowie einen Labortrakt. Das ärztliche Kollegium der Hautklinik umfasste bis zum Jahre 1958 nur 2 Assistentenstellen und ab 1951 eine zusätzliche Oberarztstelle.

Von 1956-1964 bekleidete Norbert Klüken diese Oberarztfunktion und habilitierte 1957 als erster Dermatologe während des Direktorats von Rudolf Strempel mit einer Arbeit „Über akrale Gefäßreaktionen“. Klüken übernahm 1964 den Aufbau der zunächst mit der Hautklinik verbundenen Angiologischen Abteilung an der Universität Essen und wurde später dort Direktor der eigenständigen Klinik und Poliklinik für Angiologie und Phlebologie. Als Gründungspräsident der Deutschen Gesellschaft für Angiologie (1965) und Vorstandsmitglied der noch jungen Deutschen Gesellschaft für Phlebologie gehört Klüken zu den Protagonisten der dermatologischen Angiologie [6-8]. 


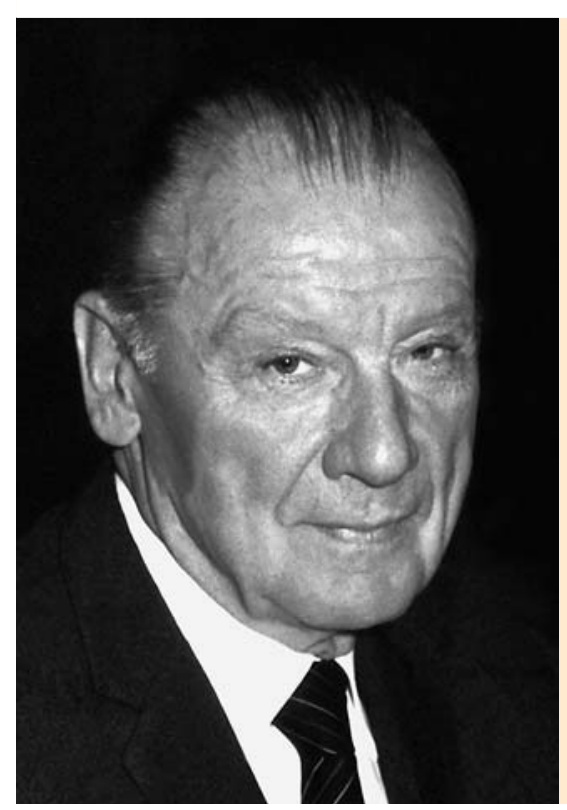

Abb. 6 Fritz Nödl (1912-1994): Zweiter Ordinarius der Homburger Universitäts-Hautklinik, wirkte von $1960-1980$.

\section{Ära Prof. Nödl (1960 - 1980)}

Als Nachfolger von Rudolf Strempel übernahm im Sommersemester 1960 Professor Fritz Nödl (1912-1994) ( Abb.6) die Leitung der Universitäts-Hautklinik.

Geboren in Brüx (Sudetenland) erhielt er seine dermatologische Ausbildung an den Universitäts-Hautkliniken in Prag und später in Breslau. Somit war Nödl in den Jahren vor dem Zweiten Weltkrieg Schüler von Heinrich Gottron, der zu diesem Zeitpunkt die Breslauer Hautklinik leitete und damals als „ungekrönter König der Dermatologie“ angesehen wurde.

Während des Krieges diente Nödl als Regimentsarzt bei den Fallschirmspringern (sportliches Fallschirmspringen soll bis ins hohe Alter seine Leidenschaft geblieben sein). 1947 konnte er sich wieder dem Fach Dermatologie widmen und wurde Oberarzt unter Horst-Günther Bode an der Universitäts-Hautklinik Göttingen, wo er sich 1950 mit einer Arbeit über den Morbus Kaposi habilitierte. Neben seiner wissenschaftlichen Forschungsarbeit über Basaliome, neurogene Tumoren, Strahlenwirkungen an der Haut, krankhaftem Haarausfall sowie über das maligne Melanom und das Problem der lymphogenen Metastasierung, galt er als einer der besten Dermatohistologen seiner Zeit.

Verglichen mit der damals hochmodernen Göttinger Klinik war die Homburger Hautklinik sehr bescheiden ausgestattet. Nödl soll es als Herausforderung angesehen haben, die Homburger Hautklinik dem ihm gewohnten Standard anzupassen. Innerhalb kurzer Zeit nach Übernahme der Klinik gelang ihm die Einrichtung eines Histologischen Labors und einer Poliklinik. Daneben verdanken wir ihm die Einrichtung eines mykologischen Labors, einer Abteilung für Strahlentherapie und die Etablierung der Allergologie in der Hautklinik. Zu erwähnen bleiben dabei auch die durch ihn erreichten besseren Bedingungen für Patienten und Personal durch Umbau der Krankensäle in überschaubare Krankenzimmer sowie durch Schaffung von Sozialräumen und den Anbau von Aufzügen [6-8].

Gefördert wurde der Ausbau sicherlich durch den generellen Innovationsschub der Universitätskliniken in den 70er-Jahren, währenddessen es zur Aufteilung von größeren Kliniken in mehrere Abteilungen bzw. zu Neugründungen kam, was sich nur mit entsprechenden Baumaßnahmen und Geräteausstattungen ver- wirklichen ließ. Diese Bautätigkeit war wiederum gefördert durch das am 1.9. 1969 beschlossene „Hochschulbauförderungsgesetz (HBFG)“, das Bund und Länder zu gleichen Anteilen zur Übernahme der Kosten für Hochschulbau und Großgeräte verpflichtete $[4,5]$.

Neben dem Ausbau der Hautklinik konnte auch deren ärztliches Kollegium erweitert werden. Dabei kam 1961 Albert Schimpf neben Norbert Klüken als zweiter Oberarzt hinzu. Habilitiert 1960 in Leipzig mit einer Arbeit über die Pathogenese des Berufsekzems, ist Albert Schimpf die Einrichtung eines leistungsfähigen Labors für Allergologie- und Hautfunktionsdiagnostik an der Homburger Hautklinik zu verdanken und damit war auch der erste Schritt in Richtung des Schwerpunktes der Berufsdermatologie erfolgt. Insgesamt verlagerte sich das stationär behandelte Patientengut in den 60er-Jahren von den Infektionskrankheiten hin zu den chronisch entzündlichen Erkrankungen der Haut [6 -8].

\section{Ära Prof. Zaun (1980 - 1996)}

$\nabla$

Die Leitung der Hautklinik wurde im Wintersemester 1980/81 von Nödl an seinen ehemaligen Assistenz- und späteren Oberarzt Professor Hansotto Zaun (geb. 1930) übergeben ( $\boldsymbol{Q}$ Abb. 7).

Nach Examen und Promotion wechselte Zaun 1958 von Marburg als Medizinalassistent an die Göttinger Hautklinik, wo Nödl noch als Oberarzt tätig war. 1962 folgte Zaun dann nach Homburg und wurde, nachdem Klüken 1964 die Homburger Hautklinik verließ, Oberarzt unter Nödl. Er habilitierte sich 1967 mit einer Arbeit über Pathologische Reaktionen am Haarfollikel als zweiter Dermatologe in Homburg. Ziele seiner Forschungstätigkeit waren die Pathologie des Haarfollikels, die Zusammenhänge zwischen Magenfunktion und Haut, Endokrinium und Haut sowie die Wirkung von Kortikosteroiden auf Blutgefäße. Sein damaliger Doktorand Peter Altmeyer, heute Direktor der Klinik für Dermatologie der Ruhr-Universität Bochum, war dabei maßgeblich an der Erforschung der Wirkung von Steroiden auf die Haut beteiligt. 1974 verließ Zaun zunächst die Homburger Hautklinik und übernahm die Leitung der Städtischen Hautklinik Bremerhaven, hielt aber weiterhin regelmäßig Vorlesungen in Homburg und kehrte 1980 als Ordinarius an die Homburger Hautklinik zurück. In den Berufungsverhandlungen erreichte er eine weitere Erhöhung der Mitarbeiterzahl und Mittelzusagen zur Neueinrichtung von

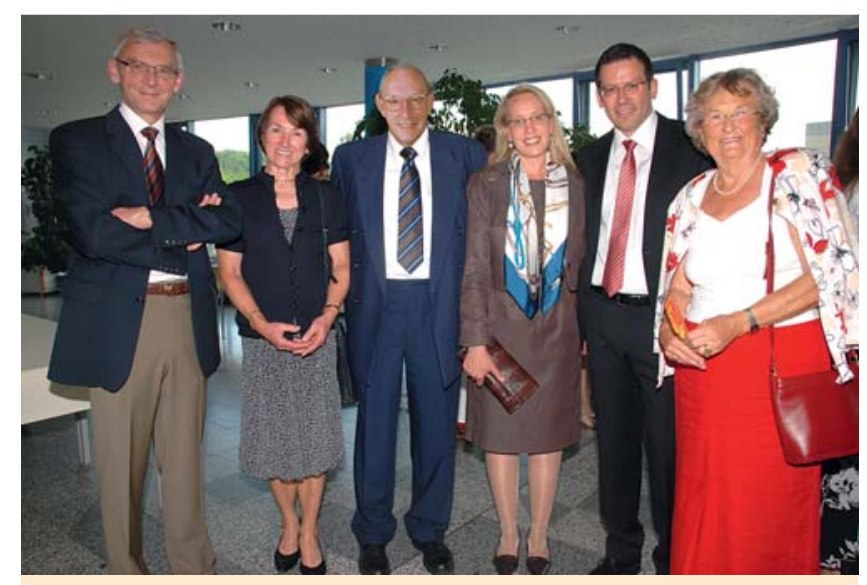

Abb. 7 Hansotto Zaun (Jg. 1930). Dritter Ordinarius der Homburger Universitäts-Hautklinik (1980 - 1996) in Mitten Ehepaar Landthaler (li), Vogt (re) und seiner Gattin ganz rechts anlässlich der Antrittsvorlesung Prof. Vogts am 30. Juni 2010. 
Tab. 2 Oberärzte der Universitäts-Hautklinik (1980 - 2010).

\begin{tabular}{|l|l|}
\hline Friedrich Bahmer & $(1981-1994)$ \\
\hline Monika Kegel & $(1983-1989)$ \\
\hline Heidelore Hofmann & $(1986-1992)$ \\
\hline Hans-Peter Baum & $(1992-1997)$ \\
\hline Patrick Koch & $(1992-2006)$ \\
\hline Dorothee Dill-Müller & $(1997-2008)$ \\
\hline Uwe Reinhold & (seit 2000) \\
\hline Jörg Reichrath & (seit 2003) \\
\hline Knuth Rass & (seit 2005) \\
\hline Claudia Pföhler & (seit 2008) \\
\hline Hortensia Schimpf & (seit 2009) \\
\hline Cornelia Müller & (seit 2010) \\
\hline Alexander Rösch &
\end{tabular}

Laboren, welche durch Ausbau von Kellern und Speichern der Klinik Platz fanden. Die Weiterentwicklung der Hautklinik unter seiner Leitung, insbesondere im Bereich der Forschung und Erweiterung des therapeutischen Repertoirs, ist sicherlich auch einer Reihe engagierter und kompetenter Mitarbeiter ( Tab. 2), unter ihnen insbesondere Professor Friedrich Bahmer (heute Direktor der Klinik für Dermatologie und Allergologie in Bremen), zu verdanken.

Forschungsschwerpunkte waren Hormonwirkungen auf Talgdrüsen und Haarfollikel, Evaluierung von Spermienfunktionstests, quantitative Histologie, Mechanismen der Tumormetastasierung, Expression von Adhäsionsmolekülen auf Hauttumoren, molekulare Steuermechanismen des Haarwachstums, Allergien auf Amalgam und Schwermetalle, molekulare Grundlagen der therapeutischen Wirkungen von 1,25 Dihydroxy-Vitamin D3 und Retinsäure in der Haut sowie der Vitamin D-Stoffwechsel bei Psoriasis. Unter Zauns Leitung konnte die Hautklinik über 50 Dissertationen und 400 wissenschaftliche Publikationen verzeichnen. Zu erwähnen sind als Neuerung ferner die regelmäßig stattfindenden Fortbildungsnachmittage der Klinik gemeinsam mit dem Berufsverband (Landesstellen Rheinland-Pfalz und Saar) und Seminartagungen mit dem Ziel einer besseren Kooperation von Klinikern und Praktikern [6-8].

Während der Amtszeit Zauns wurde der Krankenhausbetrieb der Universitätskliniken auf massives Drängen des Wissenschaftsministers des Saarlandes 1990 letztendlich dem Wissenschaftsministerium unterstellt, sodass 1994 die Universitätskliniken in Gänze als „Universitätskliniken des Saarlandes“ in die Universität des Saarlandes integriert werden konnten $[4,5]$.

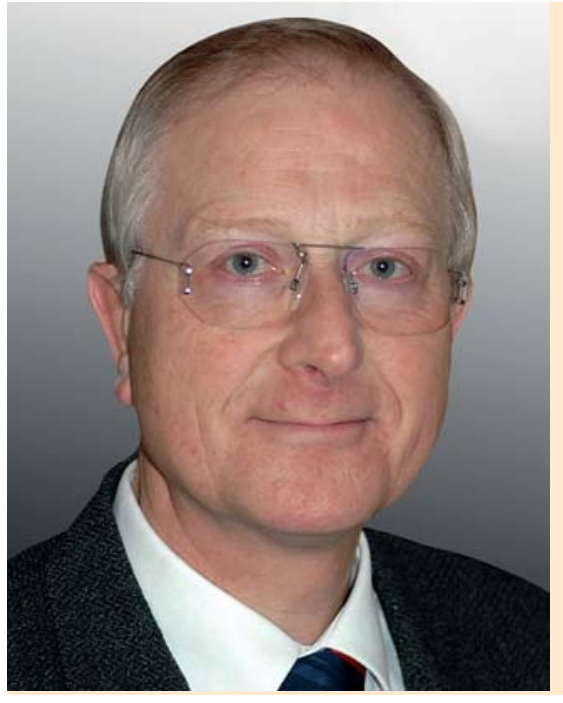

Abb. 8 Wolfgang Tilgen. Vierter Ordinarius der Homburger Universitäts-Hautklinik (1996-2009).

\section{Ära Prof. Tilgen (1996-2009)} $\nabla$

Am 17. Juni 1996 nahm Prof. Wolfgang Tilgen (geb. 1944) als neu berufener Ordinarius der Haut- und Poliklinik der Universitätskliniken des Saarlandes seine Tätigkeit auf ( $\bullet$ Abb. 8).

Als Schüler von Urs Schnyder und Detlef Petzoldt absolvierte Tilgen seine dermatologische Facharztweiterbildung an der Heidelberger Universitäts-Hautklinik, wurde dort Oberarzt und habilitierte sich 1989 zur Charakterisierung maligner Hauttumoren in vivo und in vitro. Im Zuge der Berufungsverhandlungen konnte er die ehemalige operative Station der Frauenklinik mit großzügigen Operationsräumen als Hauptgebäude der Homburger Hautklinik übernehmen ( $\bullet$ Abb. 9).

Die dortige Installation des Haut-OPs mit angeschlossener operativer Station und einer neuen Onkologischen Ambulanz reflektierte bereits die klinischen Schwerpunkte, die Ausrichtung und Entwicklung der Hautklinik unter Tilgen.

Als Vorstandsmitglied der „Arbeitsgemeinschaft Dermatologische Onkologie“ (ADO) der Deutschen Dermatologischen Gesellschaft (1994-2000) und Vorstandsmitglied der Deutschen Krebsgesellschaft (2002 - 2008) forcierte er die Dermato-Onkologie in Homburg z.B. durch die Etablierung einer systematischen Nachsorgesprechstunde für Hauttumorpatienten und den Ausbau der Hautklinik als Studienzentrum der ADO und für Arzneimittelstudien insbesondere für Patienten mit malignem Melanom.

Das operative Spektrum wurde in diesen Jahren u.a. durch die Aufnahme der Schildwächterlymphknoten-Biopsie und der Phlebochirurgie maßgeblich erweitert. Neue diagnostische Verfah-
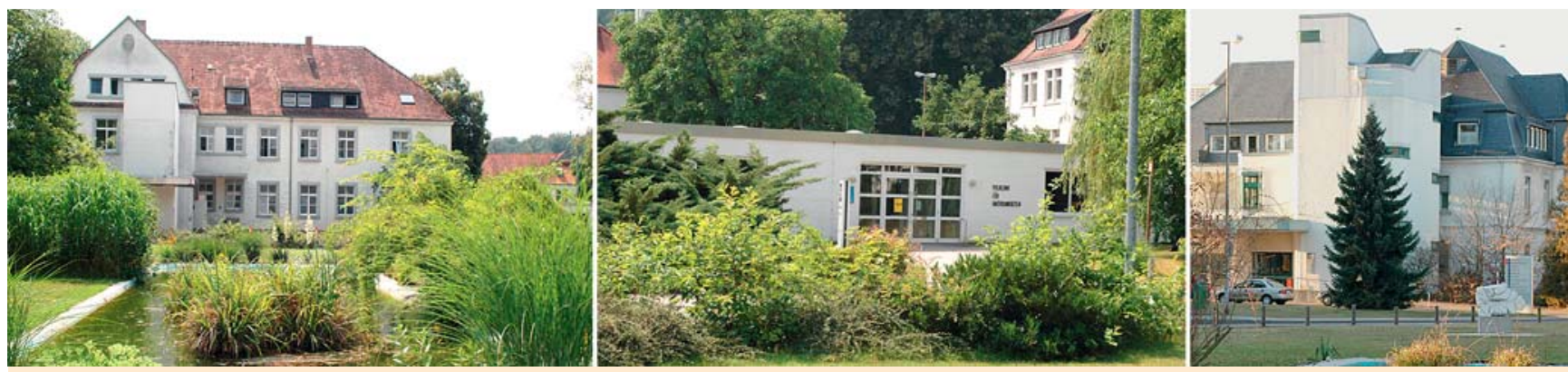

Abb. 9 Aktuelles Gesicht der Klinik. Die Klinik für Dermatologie ist aktuell noch an 3 Standorten in den Gebäuden 35/36 und 18 untergebracht. Ein Umzug und teilweiser Neubau ist für 2011 - 12 geplant. 


\begin{tabular}{|c|c|}
\hline 3. - 4. Oktober 1970 & $\begin{array}{l}\text { 99. Tagung der Vereinigung Südwest- } \\
\text { deutscher Dermatologen }\end{array}$ \\
\hline 4. -6. Mai 1990 & $\begin{array}{l}\text { 120. Tagung der Vereinigung Südwest- } \\
\text { deutscher Dermatologen (Saarbrücken) }\end{array}$ \\
\hline 2. - 3. Oktober 1998 & $\begin{array}{l}\text { 8. Jahrestagung der Arbeitsgemein- } \\
\text { schaft Dermatologische Onkologie }\end{array}$ \\
\hline 17. - 18. September 1999 & $\begin{array}{l}\text { 1. Internationales Symposium „Minimal } \\
\text { Residual Disease in Melanoma: Biology, } \\
\text { Detection and Clinical Relevance of } \\
\text { Micrometastases“ }\end{array}$ \\
\hline 8. - 9. Oktober 1999 & $\begin{array}{l}\text { 128. Jahrestagung der Vereinigung } \\
\text { Südwestdeutscher Dermatologen }\end{array}$ \\
\hline 14. - 15. September 2001 & $\begin{array}{l}\text { 1. Internationales Symposium der Inter- } \\
\text { national Society for Chemosensitivity } \\
\text { Testing in Oncology }\end{array}$ \\
\hline 3. - 4. Mai 2002 & $\begin{array}{l}\text { 1. Internationales Symposium „Vitamin } \\
\text { D Analogs in Cancer Prevention and } \\
\text { Therapy“ }\end{array}$ \\
\hline 7. - 8. Mai 2005 & $\begin{array}{l}\text { 2. Internationales Symposium „Vitamin } \\
\text { D Analogs in Cancer Prevention and } \\
\text { Therapy“ (Homburg/Lübeck) }\end{array}$ \\
\hline 6. - 7. Juni 2008 & $\begin{array}{l}\text { 4. Strategiesitzung der Deutschen } \\
\text { Gesellschaft für Dermatochirurgie }\end{array}$ \\
\hline
\end{tabular}

ren, wie die digitale Auflichtmikroskopie oder die Sonografie von Haut und Lymphknoten und therapeutische Innovationen, wie die fotodynamische Therapie, die endoluminale Lasertherapie der Varikosis und die extrakorporale Fotopherese wurden implementiert bzw. ausgebaut.

Die 13-jährige Amtszeit Tilgens war auch gekennzeichnet durch eine Vielzahl nationaler und internationaler Kongresse ( Tab. 3), die die Forschungsschwerpunkte der Klinik repräsentierten.

Bereits 1998 richtete die Homburger Hautklinik die 8. Jahrestagung der ADO aus. Es folgten von 1999 bis 2002 international hochkarätig besetzte Kongresse zur Mikrometastasierung des malignen Melanoms und zur Chemosensitivitätstestung jeweils unter der Leitung von Uwe Reinhold, den Tilgen 1997 als leitenden Oberarzt von Bonn nach Homburg holte, sowie zur Bedeutung von Vitamin D-Analoga auf dem Gebiet der Onkologie unter der Leitung von Jörg Reichrath, der im Jahr 2000 über dieses Thema in Homburg habilitierte. Darüber hinaus war die Homburger Hautklinik durch den Vorsitz der Saarländischen Krebsgesellschaft, den Tilgen von 2000-2007 innehatte, in diesem Zeitraum mehrfach an der Ausrichtung des Saarländischen Krebskongresses beteiligt.

Unter dem Ordinariat von Prof. Tilgen wurde die klinische Kompetenz der Homburger Hautklinik auch durch den Erwerb der Weiterbildungsbefugnisse für Phlebologie, medikamentöse Tumortherapie und Dermatohistologie deutlich gestärkt. Zum Ende seiner Amtszeit wurden noch Pläne zum Ausbau der Dermatohistologie aufgenommen, die sein Nachfolger dann in die Tat umsetzte.

\section{Gegenwart und Ausblick \\ $\nabla$}

Zum 1. Dezember 2009 wurde Herr Prof. Dr. med. Thomas Vogt auf den Lehrstuhl für Dermatologie und Venerologie des Saarlandes berufen ( $\bullet$ Abb. 10).

Professor Vogts Ausbildung wurde geprägt von international renommierten Dermatologen wie Prof. Dr. Dr. h.c. mult. Otto

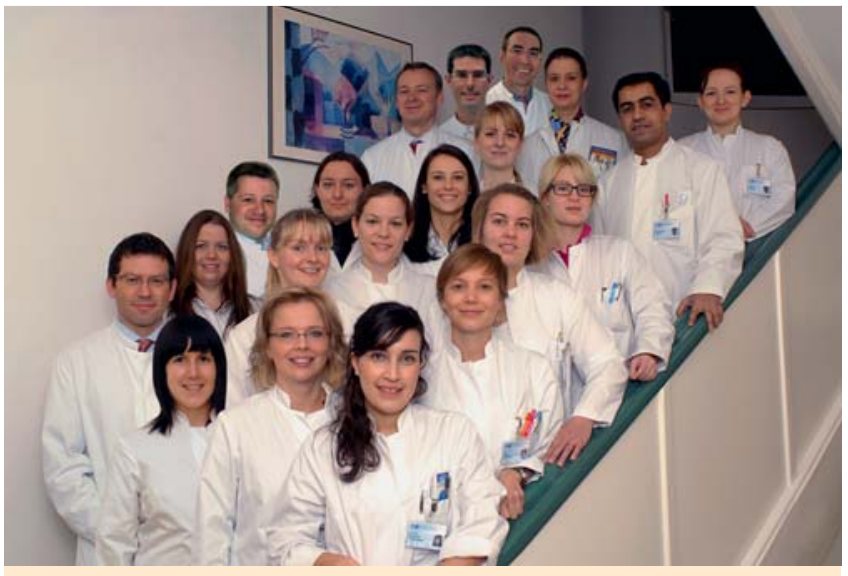

Abb. 10 Ärztliches Kollegium der Klinik für Dermatologie Homburg, November 2010

Braun-Falco (em. Ordinarius der LMU München - einem gebürtigen Saarländer!), Prof. Dr. Günther Burg (em. Ordinarius Würzburg/Zürich, ehemals zeitweilig Assistenzarzt an der Homburger Hautklinik), Prof. Dr. Wilhelm Stolz (akt. Direktor der Hautklinik München Schwabing) und vor allem - über 15 Jahre - von Prof. Dr. med. Dr. h.c. Michael Landthaler (Ordinarius Univ. Regensburg). Forschungsaufenthalte in der Mitte der 90er-Jahre am Sydney Kimmel Cancer Center und am California Institute for Biological Research, CA, USA, waren die methodisch-wissenschaftlich prägenden Abschnitte im Werdegang Vogts zielend auf die molekulare Krebsforschung. Diese brachten zudem grundlegende Entdeckungen (RBBP2H1/JARID1B) für die aktuell fortgeführte Forschungsrichtung in Homburg zu JARID1B/Melanomstammzellen (siehe unten).

Aktuelle klinische Schwerpunkte unter Prof. Vogt sind die Dermato-Histologie und Dermato-Onkologie mit allen Optionen der medikamentösen Tumortherapie einschließlich extrakorporaler Fotopherese, die operative Dermatologie einschließlich innovativer endoluminaler Therapieverfahren der Varikosis sowie die Allergologie, Berufsdermatologie und Umweltmedizin.

Es konnte bereits zu Jahresbeginn eine enge dermato-histologische Kooperation mit Luxemburg (Laboratoire National de Santé) etabliert werden, was der weiteren Entfaltung der spezifischen Expertise in diesem Gebiet dienlich sein wird und bereits vermehrt Patienten aus dem Nachbarland zur Behandlung nach Homburg führt. Auch die Interaktion mit den niedergelassenen Kollegen und Zuweisern steht im Fokus einerseits durch attraktive Fortbildungsangebote (drei Veranstaltungen allein im laufenden Jahr), andererseits durch intensivierte Zusammenarbeit in der Weiterbildung junger Kolleginnen und Kollegen mit den Praxen im Lande sowie gemeinsame prozessorientierte Arbeit zur Verbesserung der Abläufe gerade im Bereich der Versorgung von Hautkrebspatienten.

Die weiteren Entwicklungen zielen auf die Etablierung eines interdisziplinären Allergiezentrums gemeinsam mit den Kliniken für Pädiatrie, Pulmologie, Arbeitsmedizin, HNO und Gastroenterologie sowie die Gründung eines Handekzemzentrums in enger Assoziation mit der Berufsdermatologie an diesem Standort. Wissenschaftlich ist es ein vorrangiges Ziel der Hautklinik, vorangetragen durch den leitenden Oberarzt der Klinik, Prof.Jörg Reichrath, den Einfluss des endokrinen Vitamin D-Systems u.a. auf die Hautkrebsentstehung (siehe Minireview in dieser Ausgabe) zu erforschen. Im Mai 2011 wird es in Homburg einen zweiten internationalen Kongress zur Vitamin D-Forschung, gefördert durch die 
Deutsche Forschungsgemeinschaft, DFG, geben ( $4^{\text {th }}$ International Symposium: Vitamin D and Analogs in Cancer Prevention and Therapy 20-21 Mai 2011, Schlossberg-Hotel Homburg). Auch wurden aktuell zwei neue Forschungsvorhaben definiert und befinden sich in der Antragsphase. Im Zuge der Neuberufung von Prof. Vogt stehen erhebliche investive Mittel der Universität und Fakultät zur Verfügung neben weiteren Drittmitteln (ca. 350000 EUR), um die Laborgeräteausstattung in der Hautklinik weiter abzurunden. Die Stärke des Standorts ist jedoch neben einem räumlich und technisch zureichenden Labor in der Hautklinik vor allem die Vernetzung und unkomplizierte Zusammenarbeit mit den Partnern in anderen Kliniken, dem José Carreras-Zentrum, dem Institut für experimentelle Chirurgie und den Instituten der theoretischen und vorklinischen Fächer mit einem SFBSchwerpunkt im Bereich der Erforschung membrannaher Prozesse und Ionenkanäle. Die Hautklinik verfolgt auch hier in Kooperation innovative Projekte im Bereich der Erforschung der CalciumIonenkanäle in Melanomzellen. Unter diesen günstigen Voraussetzungen war es bereits im zu Ende gehenden Jahr möglich auch ein ambitioniertes DFG-gefördertes Projekt zur Melanomforschung (siehe Minireview zur Melanomstammzelle in dieser Ausgabe) zu initiieren und aus den USA vom renommierten WistarInstitut in Philadelphia Herrn PD Alexander Rösch an die Hautklinik der Universität des Saarlandes zu holen.

Das Team ist somit aktuell auf fünf Oberärzte und 12 Assistenten sowie vier Gastärzte bzw. Gastwissenschaftler angewachsen. Drei Kollegen befinden sich auf „Habilitationskurs“, die Lehre wurde in diesem Jahr exzellent evaluiert, zahlreiche Doktoranden rekrutiert, nahezu alle PJ-Studenten der Hautklinik wollen auch hier ihre Ausbildung fortsetzen und fühlen sich wohl. Die Assistenten waren in den Weiterbildungsgesprächen durchweg motiviert und sehr zufrieden. Der Nachwuchs an Jungdermatologen scheint für die Region auf einem guten Weg zu sein.

\section{Abstract}

\section{Development of the Department of Dermatology at the Saarland University Hospital \\ $\nabla$}

In 2009 the Saarland University Hospital was celebrating its $100^{\text {th }}$ anniversary. In 1945 already a department for dermatology and venerology was established, which in 1947 offered the second largest number of carrying capacity after the clinic for internal medicine. Today the Clinic for Dermatology, Venerology and Allergology possesses a modern and widespread infrastructure of a hospital for maximal care. This paper should deliver insight into foundation and the most important milestones in the development of the Clinic from its foundation till this day.

\section{Literatur}

1 Stadtarchiv Homburg. Programm, Erläuterungsbericht und Kostenvoranschlag für den Neubau einer II. Pfälz. Kreisirrenanstalt bei Homburg, 1904. Erläuterungsbericht S. 1-2. Sitzungen des Stadtrates $1903-$ 1922. Auswertung der Homburger Tagespresse.

2 Tascher G. „Entwicklung des Gesundheitswesens im Saargebiet und Saarland von 1920-1956 im Spiegel der machtpolitischen Verhältnisse“, erweiterte Fassung der Promotionsarbeit 2007 am Institut für Geschichte der Medizin der Ruprecht-Karls-Universität Heidelberg Referent Prof. Dr. W.U. Eckart

3 Stadtarchiv Homburg, Saarländisches Landesarchiv Saarbrücken; Krankenbuchlager Berlin, Bayerisches Staatsarchiv Abtl. IV Kriegsarchiv; Militärarchiv (Bundesarchiv Freiburg; Dr. Klaus Doenecke. „Die Anstalt“" (unveröffentlichtes Manuskript) und persönliche Mitteilung

4 Denkschrift zur Errichtung der Pfälzischen Heil- und Pflegeanstalt Homburg. Speyer: Verlag der Kgl. Regierung der Pfalz; 1910: 38 - 39

5 Sonderausgabe 60 Jahre UKS 1947 - 2007, UKS Report, Homburg: April 2007

6 Zaun H. Die Homburger Hautklinik: 50 Jahre Universitätsklinik. Hautarzt 1998; 49: 317-321

7 Zaun H. Zur Geschichte der Medizinischen Fakultät und der Hautklinik in Homburg. Berlin: Grosse; Programmheft: 22. Forbildungsseminar d. Berufsverb. Dtsch Dermatol 1983: 6-11

8 Zaun H. 50 Jahre Hochschuldermatologie in Homburg. Saarländisches Ärzteblatt 1997; 9: 16-18 